\title{
OPTIMAL INFORMATION THEORETIC CAPACITY OF THE PLANAR CELLULAR UPLINK CHANNEL
}

\author{
Symeon Chatzinotas, Muhammad Ali Imran, Costas Tzaras
}

\author{
Centre for Communication Systems Research, University of Surrey, United Kingdom, GU2 7XH
}

\begin{abstract}
The majority of information-theoretic hyper-receiver cellular models preserve a fundamental assumption which has initially appeared in Wyner's [1] model, namely the collocation of User Terminals (UTs). Although this assumption produces more tractable mathematical models, it is unrealistic with respect to current practical cellular systems. In this paper, we alleviate this assumption by assuming uniformly distributed UTs. The model under investigation is a Gaussian Cellular Multiple Access Channel (GCMAC) over a planar cellular array in the presence of power-law path loss and flat fading. In this context, we evaluate the effect of UT distribution on the optimal sum-rate capacity by considering a variable-density cellular system. Furthermore, we compare the sum-rate capacity produced by the planar and the linear cellular array. Finally, the analytical results are interpreted in the context of a typical macrocellular scenario.
\end{abstract}

\section{INTRODUCTION}

The first concrete result for the information-theoretic capacity of the Gaussian Cellular Multiple Access Channel (GCMAC) was presented by Wyner in [1]. Using a very simple but tractable model for the cellular uplink channel, Wyner showed the importance of joint decoding at the Base Station (BS) receivers (hyper-receiver) and found the analytical formulas of the maximum system capacity. This model triggered the interest of the research community in the cellular capacity limits and was extended in [2] to include flat fading environments. One major assumption shared in these models was that the cell density is fixed and only physically adjacent cells interfere. The author in [3], extended the model by assuming multiple-tier interference and incorporated a distancedependent path loss factor in order to study the effect of cell density in a linear cellular array. However, the assumption

The work reported in this paper has formed part of the "Fundamental Limits to Wireless Network Capacity" Elective Research Programme of the Virtual Centre of Excellence in Mobile \& Personal Communications, Mobile VCE, www.mobilevce.com. This research has been funded by the following Industrial Companies who are Members of Mobile VCE - BBC, BT, Huawei, Nokia, Nokia Siemens Networks, Nortel, Vodafone. Fully detailed technical reports on this research are available to staff from these Industrial Members of Mobile VCE. The authors would like to thank Prof. G. Caire and Prof. D. Tse for the useful discussions. of collocation of all UTs in each cell was maintained to keep the model tractable. In this paper, we extend these models in order to incorporate the effect of user distribution. Instead of assuming collocated UTs, we assume that UTs are spatially distributed within the cell and each channel gain is affected by a distance-dependent path loss factor. The rest of the paper is organised as follows. In the next section, we describe the proposed model and we describe the derivation of the information theoretic capacity of the cellular system. In section 3 , we evaluate and compare the capacity results produced by both simulation and analysis. In addition, section 4 interprets the analytical results in the context of a typical macrocellular scenario. The last section concludes the paper.

\section{MODEL DESCRIPTION AND ANALYSIS}

Assume that the $K$ users are uniformly distributed in each cell of a planar cellular system comprising $N$ base stations. Assuming flat fading, the received signal at cell $n$, at time index $t$, will be given by:

$$
y^{n}[t]=\sum_{m=1}^{N} \sum_{k=1}^{K} \varsigma_{k}^{n m} g_{k}^{n m}[t] x_{k}^{m}[t]+z^{n}[t]
$$

where $x_{k}^{m}[t]$ is the $t$ th complex channel symbol transmitted by the $k$ th UT of the $m$ th cell and $\left\{g_{k}^{n m}\right\}$ are independent, strictly stationary and ergodic complex random processes in the time index $t$, which represent the flat fading processes experienced in the transmission path between the $n$th BS and the $k$ th UT in the $m$ th cell. The fading coefficients are assumed to have unit power, i.e. $\mathbb{E}\left[\left|g_{k}^{n m}[t]\right|^{2}\right]=1$ for all $(n, m, k)$ and all UTs are subject to an average power constraint, i.e. $\mathbb{E}\left[\left|x_{k}^{m}[t]\right|^{2}\right] \leq P$ for each $(m, k)$. The interference factors $\varsigma_{k}^{n m}$ in the transmission path between the $m$ th BS and the $k$ th UT in the $n$th cell are calculated according to the "modified" power-law path loss model $[3,4]: \varsigma_{k}^{n m}=\left(1+d_{k}^{n m}\right)^{-\eta / 2}$, where $\eta$ is the path loss exponent. Dropping the time index $t$, the aforementioned model can be more compactly expressed as a vector memoryless channel of the form $\mathbf{y}=\mathbf{H x}+\mathbf{z}$, where the vector $\mathbf{y}=\left[y^{1} \ldots y^{N}\right]^{T}$ represents received signals by the BSs, the vector $\mathbf{x}=\left[x_{1}^{1} \ldots x_{K}^{N}\right]^{T}$ represents transmit signals by all the UTs of the cellular system and the components of vector $\mathbf{z}=\left[z^{1} \ldots z^{N}\right]^{T}$ are i.i.d c.c.s. random variables 
representing AWGN with $\mathbb{E}\left[z^{n}\right]=0, \mathbb{E}\left[\left|z^{n}\right|^{2}\right]=\sigma^{2}$. The channel matrix $\mathbf{H}$ can be written as $\mathbf{H}=\boldsymbol{\Sigma} \odot \mathbf{G}$, where $\boldsymbol{\Sigma}$ is a $N \times K N$ deterministic matrix and $\mathbf{G} \sim \mathcal{C N}(\mathbf{0}, \mathbf{I})$ is a complex Gaussian $N \times K N$ matrix, comprising the corresponding Rayleigh fading coefficients. The entries of the $\Sigma$ matrix are defined by the variance profile function

$$
\varsigma(u, v)=(1+d(u, v))^{-\eta / 2},
$$

where $u \in[0,1]$ and $v \in[0, K]$ are the normalized indexes for the BSs and the UTs respectively and $d(u, v)$ is the normalized distance between BS $u$ and UT $v$. According to [5], the asymptotic sum-rate capacity $C_{\text {opt }}$ for this model assuming a very large number of cells, is given by

$$
\begin{aligned}
C_{\mathrm{opt}} & =\lim _{N \rightarrow \infty} \frac{1}{N} \mathcal{I}(\mathbf{x} ; \mathbf{y} \mid \mathbf{H}) \\
& =\lim _{N \rightarrow \infty} \mathbb{E}\left[\frac{1}{\mathrm{~N}} \sum_{\mathrm{i}=1}^{\mathrm{N}} \log \left(1+\frac{\tilde{\gamma}}{K} \lambda_{i}\left(\frac{1}{N} \mathbf{H H}^{\dagger}\right)\right)\right] \\
& =\int_{0}^{\infty} \log \left(1+\frac{\tilde{\gamma}}{K} x\right) d \mathrm{~F}_{\frac{1}{N} \mathbf{H} \mathbf{H}^{\dagger}}(x) \\
& =\mathcal{V}_{\frac{1}{N} \mathbf{H} \mathbf{H}^{\dagger}}(\tilde{\gamma} / K)=K \mathcal{V}_{\frac{1}{N} \mathbf{H}^{\dagger} \mathbf{H}}(\tilde{\gamma} / K)
\end{aligned}
$$

where $\tilde{\gamma}=K N P / \sigma^{2}=K N \hat{\gamma}$ is the system transmit power normalized by the receiver noise power $\sigma^{2}$. The term $\lambda_{i}(\mathbf{X})$ denotes the eigenvalues of matrix $\mathbf{X}$ and

$$
\begin{aligned}
\mathcal{V}_{\mathbf{X}}(\gamma) & \triangleq \mathbb{E}\left[\log \left(1+\gamma \lambda_{i}(\mathbf{X})\right)\right] \\
& =\int_{0}^{\infty} \log \left(1+\gamma \lambda_{i}(\mathbf{X})\right) d \mathbf{F}_{\mathbf{X}}(x)
\end{aligned}
$$

is the Shannon transform with parameter $\gamma$ of a random square Hermitian matrix $\mathbf{X}$, where $\mathrm{F}_{\mathbf{X}}(x)$ is the cumulative function of the asymptotic eigenvalue distribution (a.e.d.) of matrix $\mathbf{X}$ [5]. For a rectangular Gaussian matrix $\mathbf{G} \sim \mathcal{C N}(\mathbf{0}, \mathbf{I})$ with $\beta$ being the columns/rows ratio, the a.e.d. of $\frac{1}{N} \mathbf{G}^{\dagger} \mathbf{G}$ converges almost surely (a.s.) to the nonrandom a.e.d. of the MarčenkoPastur law

$$
\mathcal{V}_{\frac{1}{N} \mathbf{G}^{\dagger} \mathbf{G}}(\gamma) \stackrel{a . s .}{\longrightarrow} \mathcal{V}_{\mathrm{MP}}(\gamma, \beta)
$$

where $\mathcal{V}_{\mathrm{MP}}(\gamma, \beta)=\log \left(1+\gamma-\frac{1}{4} \phi(\gamma, \beta)\right)$

$$
+\frac{1}{\beta} \log \left(1+\gamma \beta-\frac{1}{4} \phi(\gamma, \beta)\right)-\frac{1}{4 \beta \gamma} \phi(\gamma, \beta)
$$

and $\phi(\gamma, \beta)=$

$$
\left(\sqrt{\gamma(1+\sqrt{\beta})^{2}+1}-\sqrt{\gamma(1-\sqrt{\beta})^{2}+1}\right)^{2} .
$$

However, considering the described cellular channel the channel matrix contains elements of non-uniform variance. In this case, the a.e.d. of $\frac{1}{N} \mathbf{H H}^{\dagger}$ is derived based on the analysis in
[3] and using tools from the discipline of Free Probability. In this direction, the Shannon transform can be approximated by a scaled version of the Marčenko-Pastur law

$$
\mathcal{V}_{\frac{1}{N} \mathbf{H}^{\dagger} \mathbf{H}}(\tilde{\gamma} / K) \simeq \mathcal{V}_{\mathrm{MP}}(q(\boldsymbol{\Sigma}) \tilde{\gamma} / K, K)
$$

where $q(\boldsymbol{\Sigma}) \triangleq\|\boldsymbol{\Sigma}\|^{2} / K N^{2}$ with $\|\boldsymbol{\Sigma}\|^{2} \triangleq \operatorname{tr}\left\{\boldsymbol{\Sigma}^{\dagger} \boldsymbol{\Sigma}\right\}$ being the Frobenius norm of the $\Sigma$ matrix. In the asymptotic case $q(\boldsymbol{\Sigma})$ is given by

$$
\lim _{N \rightarrow \infty} q(\boldsymbol{\Sigma})=\frac{1}{K} \int_{0}^{1} \int_{0}^{K} \varsigma^{2}(u, v) d u d v .
$$

Since the variance profile function of Equation (2) defines rectangular block-circulant matrix with $1 \times K$ blocks which is symmetric about $u=K v$, the channel matrix $\mathbf{H}$ is asymptotically row-regular and thus the asymptotic norm of $\mathbf{h}_{i}$ converges to a deterministic constant for every BS

$$
\lim _{N \rightarrow \infty} q(\boldsymbol{\Sigma})=\frac{1}{K} \int_{0}^{K} \varsigma^{2}(v) d v, \forall u \in[0,1] .
$$

According to [3], this approximation holds for UTs collocated with the BS in a linear cellular array. Herein, we show that the approximation holds for the case of distributed UTs over a planar cellular array. Furthermore, in [6] it is stated that the limiting eigenvalue distribution converges to the MarčenkoPastur law, as long as $\boldsymbol{\Sigma}$ is asymptotically doubly-regular [5, Definition 2.10]. In this paper, it is shown that on the grounds of Free Probability, the Marčenko-Pastur law can be effectively utilized in cases where $\boldsymbol{\Sigma}$ is just asymptotically rowregular.

\subsection{Structure of Variance Profile Matrix}

In order to calculate the uplink spectral efficiency analytically, a closed form for $q(\boldsymbol{\Sigma})$ is needed. The first step towards this direction is to assume that the UTs are spatially distributed on a uniform regular grid. The variance profile matrix $\boldsymbol{\Sigma}$ contains the path-loss coefficients for all the combinations of UTs and BSs of the cellular system. More specifically, each row of the matrix corresponds to a BS, whereas each column corresponds to a UT. Therefore, in order to construct a single row of the variance profile matrix, a scanning method is required, which enumerates all the UTs of the system and calculates the path-loss coefficients. This scanning method is identically repeated for all the rows/BSs until the variance profile matrix is complete. For a linear cellular array, it has been shown that this assumption can produce compact closed forms [7]. However, for a planar cellular array with distributed UTs, the selection of the appropriate scanning method is not straightforward. Furthermore, the "raster"and "zig-zag" methods employed in [2] for collocated UTs cannot be generalized for distributed UTs. On these grounds, the rest of this section presents the novel spiral scanning method introduced in this paper, which effectively tackles the UT scanning problem for 


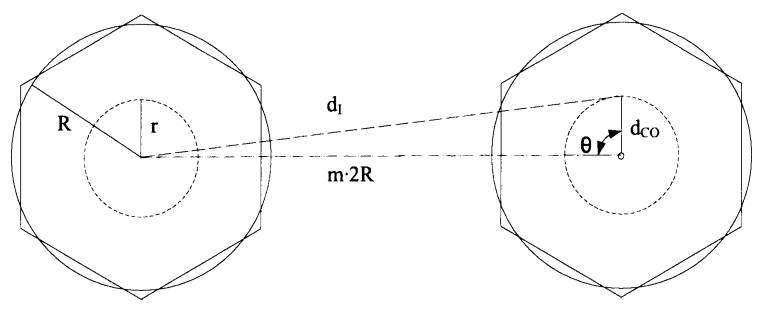

Fig. 1. Cell of interest (on the left) and cell of the $m$ th tier of interference (on the right).

a planar cellular system. In this point, it should be noted that, on the grounds of mathematical tractability, the coverage area of the hexagonal cell is approximated by the corresponding circular area of equal surface (Figure 1).

\subsubsection{Cell of interest}

Let us assume that a number of UTs lies in the coverage area of the cell of interest and it is positioned on a ring of radius $r \in[0, R]$ around the $\mathrm{BS}$, where $R$ is the cell radius (Figure 1). Since the UTs are uniformly distributed, the number of UTs positioned on the ring should increase with $r$. More specifically, the Probability Density Function (PDF) of the probability that a UT is positioned on a ring of radius $r \in[0,1]$ is given by $f(r)=2 r$ and the respective $\mathrm{Cu}-$ mulative Distribution Function (CDF) is given by $F_{r}(x)=$ $\int_{0}^{x} f(r) d r=x^{2}$. The next step is to construct a deterministic regular grid which follows this distribution function. This grid can be constructed based on the inverse CDF, namely $F_{r}^{-1}(x)=\sqrt{x}, x \in[0,1]$. Assuming that $r$ ranges in $[0, R]$ and $x \in[0, K]$ is the single-cell UT index, then the distance $d_{\mathrm{CO}}$ of the UTs positioned on the grid from the BS of interest is given by

$$
d_{\mathrm{CO}}(x)=R \sqrt{x / K} .
$$

The terms $R$ and $\sqrt{K}$ are normalization factors, coming from the fact that $K$ UTs are distributed on a cell of radius $R$. By using the normalized index $v=x / N$ instead of $x$,

$$
d_{\mathrm{CO}}(v)=R \sqrt{N / K} \sqrt{v} .
$$

\subsubsection{Cells of the mth interference tier}

In this case, the UTs that lie in a cell of the $m$ th interference tier are considered w.r.t. the BS of the cell of interest (Figure 1). According to the angular position $\theta$ of each UT in the interfering cell, its distance $d_{\mathrm{I}}^{m}$ from the BS of the cell of interest varies in $(2 m \pm 1) R$. More specifically, the distance $d_{I}^{m}$ for the $m$ th interference tier is given by the law of cosines

$$
\begin{aligned}
& d_{\mathrm{I}}^{m}(x)^{2}=(m \cdot 2 R)^{2}+d_{\mathrm{CO}}(x)^{2}-4 m R \cdot d_{\mathrm{CO}}(x) \cos \theta \\
& =(m \cdot 2 R)^{2}+\left(\frac{R}{\sqrt{K}} \sqrt{x}\right)^{2}-4 m \frac{R^{2}}{\sqrt{K}} \sqrt{x} \cos \theta .
\end{aligned}
$$

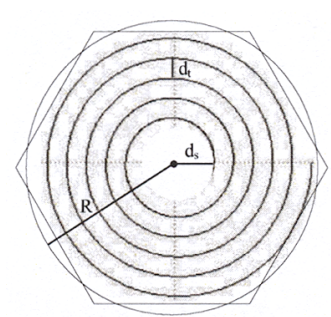

Fig. 2. Spiral scanning method

In order to drop $\theta$ in Equation (13), a spiral scanning method is employed. The locus of an Archimedean spiral using polar coordinates $\left(d_{\mathrm{CO}}, \theta\right)$ is given by

$$
d_{\mathrm{CO}}(x)=d_{s}+\left(d_{t} / 2 \pi\right) \theta
$$

where $d_{s}$ is the distance offset from the centre of the spiral and $d_{t}$ is the distance that separates successive turnings. Figure 2 depicts the path followed by the spiral scanning method in order to enumerate the UTs of a cell. In order to scan the entire cell, the distance offset has to be set to $d_{s}=0$, namely

$$
d_{\mathrm{CO}}(x)=\left(d_{t} / 2 \pi\right) \theta
$$

By combining Equations (13) and (15) and using the normalized index $v=x / N$ instead of $x$,

$$
\begin{aligned}
d_{\mathrm{I}}^{m}(v)^{2} & =(m \cdot 2 R)^{2}+\left(R \sqrt{\frac{N}{K}} \sqrt{v}\right)^{2} \\
& -4 m \cdot R^{2} \sqrt{\frac{N}{K}} \sqrt{v} \cos \left(\frac{2 \pi R}{d_{t}} \sqrt{\frac{N}{K}} \sqrt{v}\right) .
\end{aligned}
$$

In this point, it should be noted that $d_{t}$ depends on the cell radius $R$ and on the number of UTs $6 m K$ of $m$ th interfering tier and in general it should be proportional to $d_{t}(m) \propto \frac{R}{\sqrt{6 m K}}$ in order to scale accordingly to the system size.

\subsubsection{Variance profile function}

The variance profile function is utilized to calculate $q(\boldsymbol{\Sigma})$ in Equation (10). Based on the previous analysis, the variance profile function for a planar cellular array is given by

$$
\begin{aligned}
\varsigma(v) & =\varsigma_{\mathrm{CO}}(v) \sqcap\left(\frac{N t-\frac{K}{2}}{K}\right) \\
& +\sum_{m=1}^{M} \varsigma_{\mathrm{I}}^{m}\left(N t-K-\sum_{c=1}^{m-1} 6 c K\right) \\
& \sqcap\left(\frac{N t-K-\left(\sum_{c=1}^{m-1} 6 c K+3 m K\right)}{6 m K}\right) \\
& \text { where } \varsigma_{\mathrm{CO}}(v)=\left(1+d_{\mathrm{CO}}(v)\right)^{-\eta / 2}
\end{aligned}
$$




$$
\text { and } \varsigma_{\mathrm{I}}^{m}(v)=\left(1+d_{\mathrm{I}}^{m}(v)\right)^{-\eta / 2}
$$

The rect functions are used in order to apply different variance profile functions to UTs that belong to the cell of interest and to each of the $M$ interfering tiers. The factor $6 m K$ is due to the fact that the $m$ th interfering tier includes $6 m$ cells and thus $6 m K$ UTs, which can be treated equally on the grounds of symmetry.

\section{USER DISTRIBUTION RESULTS}

This section presents and compares the analytical and simulations capacity results which have been produced by applying the Free Probability approach and running Monte Carlo simulations respectively. For the analytical results, the per-cell sum-rate capacity has been calculated by combining Equations (3), (8), (10) and (17). The per-cell sum-rate capacity has been plotted w.r.t. a variable cellular system coverage. The cellular coverage is varied by varying the edge length $D$ of the cellular coverage area. The analytical results have been verified by running Monte Carlo simulations over 1000 random instances of the system and by averaging the produced capacity results. More specifically, for each system instance the Gaussian complex matrix $\mathbf{G}$ is constructed by randomly generating Gaussian i.i.d. c.c.s. fading coefficients. Similarly, the variance profile matrix $\Sigma$ is constructed by randomly placing the UTs according to the considered distribution in the coverage area of each cell and by calculating the variance profile coefficients using Equation (2). After constructing the channel matrix, the sum-rate capacity is calculated by evaluating the formula in [8]:

$$
C_{\text {opt }}=\frac{1}{N} \mathbb{E}\left[\log \operatorname{det}\left(\mathbf{I}_{N}+\hat{\gamma} \mathbf{H} \mathbf{H}^{\dagger}\right)\right] .
$$

The simulation points are marked in Figures 3-4 using circle points.

\subsection{Uniform Distribution}

Figure 3 depicts the per-cell capacity vs. the coverage area of the cellular system. The capacity for high cell densities is identical to the capacity calculated using a collocated model. However, for small cell densities the capacity keeps decreasing and for the extreme case of isolated cells, the capacity becomes zero. This phenomenon can be also intuitively explained, since in the asymptotic case, where $N$ is constant and $D \rightarrow \infty$, the distributed users of each cell will be effectively isolated from the BS of the cell.

\subsection{Cell-centre Truncated Uniform Distribution}

According to the truncated uniform distribution for cell centreusers, the UTs are uniformly distributed around the BS on a discus of radius $\rho / R$ where $\rho \in[0,1]$ is the truncation factor. The setting $\rho=0$ corresponds to the case where all the users

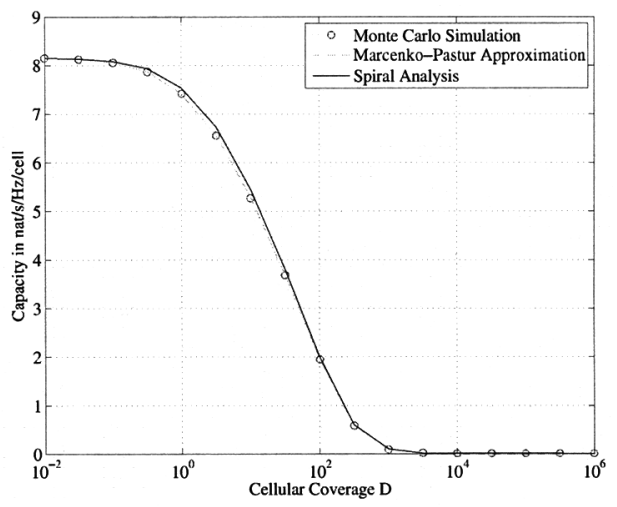

Fig. 3. Sum-rate capacity per cell $C$ (nat/s/Hz) vs. the cellular coverage $D$ for uniformly distributed users. Parameter values $N=100, \eta=2, K=4, \hat{\gamma}=10$.

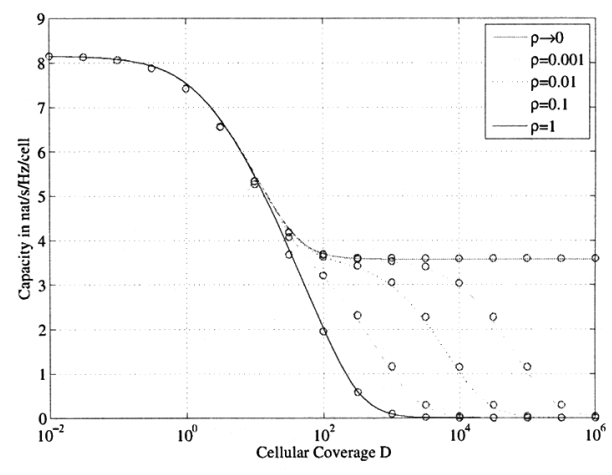

Fig. 4. Sum-rate capacity per cell $C$ (nat/s/Hz) vs. the cellular coverage $D$ for the cell-centre truncated uniform distribution. Parameter values $N=100, \eta=2, K=4, \hat{\gamma}=10$.

are collocated with the BS and the setting $\rho=1$ corresponds to the case where UTs are uniformly distributed all over the cell's coverage area. In order to produce an analytical result for this distribution, Equation (12) has to be modified as follows:

$$
d_{\mathrm{CO}}(v)=\rho R \sqrt{\frac{N}{K}} \sqrt{v}
$$

keeping the rest of the derivation as it is. Figure 4 depicts the per-cell capacity vs. the coverage area of the cellular system for the cell-centre truncated uniform distribution in the highpower regime. It can be seen that for high cell densities, the truncation has little effect on the system capacity. This is due to the fact that the variance profile gain from UTs of the cell of interest positioned closer to the BS of interest is balanced by the loss from the UTs of the interfering cells positioned further from the BS of interest. However, for small densities, the effect of the user distribution is more evident since the received power from the intra-cell UTs becomes dominant. 
Table 1. Value/Range of parameters used for a typical macrocellular scenario

\begin{tabular}{c|c|l} 
Parameter & & Value/Range \\
\hline Cell Radius & $R$ & $0.1-3 \mathrm{~km}$ \\
Reference Distance & $d_{0}$ & $1 \mathrm{~m}$ \\
Path Loss at ref. distance & $L_{0}$ & $-38 \mathrm{~dB}$ \\
Path Loss Exponent & $\eta$ & 2 usual values $\{2,3.5\}$ \\
UTs per cell & $K$ & 20 \\
UT Transmit Power & $P$ & $100-200 \mathrm{~mW}$ \\
Thermal Noise Density & $N_{0}$ & $-169 \mathrm{dBm} / \mathrm{Hz}$ \\
Channel Bandwidth & $B$ & $5 \mathrm{MHz}$ \\
\hline
\end{tabular}

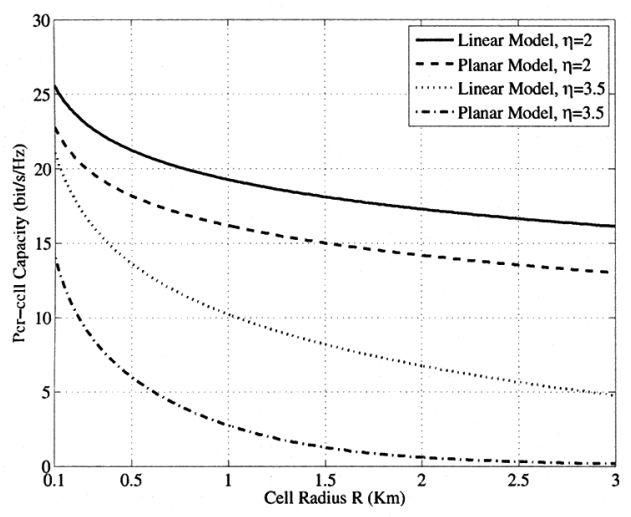

Fig. 5. Sum-rate per cell capacity (bit/s/Hz) vs. cell radius $R$ for the linear and planar cellular system. Parameters: $\eta=$ $2,3.5$ and $P=0.2 W$.

\section{PRACTICAL RESULTS}

The employed power-law path loss model of Equation (17) provides a variance profile coefficient as a function of the normalized distance $d(v)$. Similar path-loss models have been already utilized in the information-theoretic literature [3, 4]. In order to apply the aforementioned results to real-world cellular systems, a reference distance $d_{0}$ is required to interconnect the normalized distance $d(v)$ and the actual distance $\hat{d}(v)$. Assuming that the power loss at the reference distance $d_{0}$ is $L_{0}$, the scaled variance profile function is given by $\varsigma(d(v))=\left[L_{0}\left(1+\hat{d}(v) / d_{0}\right)^{-\eta}\right]^{1 / 2}$. In the context of a macro-cellular scenario, the typical parameters of Table 1 will be considered. Figure 5 depicts the per-cell capacity of the linear and the planar cellular system versus the cell radius $R$. The per-cell capacity for the linear cellular array is evaluated based on [7].

\section{CONCLUSION}

In the model employed in this paper, the assumption of collocated users in alleviated by considering users which are identically distributed in each cell and symmetrically distributed w.r.t. to each cell's BS. The analysis of this model has shown that user distribution does not affect the optimal sum-rate capacity in the high cell density regime and thus the desired results can be obtained using a planar array with collocated UTs. However, this simplification does not apply in the low cell density regime, since the interference factors of adjacent cells become insignificant and the single-cell capacity becomes dominant. From a system-design point of view, this means that the near-far effect vanishes in the context of high cell density hyper-receiver networks. In other words, the user distance from the BS of his cell becomes insignificant, since the UT's signal can be adequately received by multiple tiers of adjacent BSs.

\section{REFERENCES}

[1] A.D. Wyner, "Shannon-theoretic approach to a Gaussian cellular multiple-access channel," Information Theory, IEEE Transactions on, vol. 40, no. 6, pp. 1713-1727, Nov. 1994.

[2] O. Somekh and S. Shamai, "Shannon-theoretic approach to a Gaussian cellular multiple-access channel with fading," Information Theory, IEEE Transactions on, vol. 46, no. 4, pp. 1401-1425, July 2000.

[3] N. A. Letzepis, Gaussian Cellular Muptiple Access Channels, Ph.D. thesis, Institute for Telecommunications Research, University of South Australia, December 2005.

[4] L. Ong and M. Motani, "The capacity of the single source multiple relay single destination mesh network," in IEEE International Symposium on Information Theory, Jul 2006, pp. 1673-1677.

[5] A.M. Tulino and S. Verdu, "Random matrix theory and wireless communications," Commun. Inf. Theory, vol. 1, no. 1, pp. 1-182, 2004.

[6] A.M. Tulino, S. Verdu, and A. Lozano, "Capacity of antenna arrays with space, polarization and pattern diversity," Information Theory Workshop, 2003. Proceedings. 2003 IEEE, pp. 324-327, 31 March-4 April 2003.

[7] S. Chatzinotas, M.A. Imran, and C. Tzaras, "Information theoretic uplink capacity of the linear cellular array," in In Proc. of the Fourth Advanced International Conference on Telecommunications, AICT 2008, Athens, Greece, Jun 2008.

[8] I. E. Telatar, "Capacity of multi-antenna Gaussian channels," European Transactions on Telecommunications, vol. 10, no. 6, pp. 585-595, November 1999. 